\title{
In Silico Identification and Expression Analysis of Nuclear Factor Y (Nf-Y) Transcription Factors in Cucumber
}

\author{
Lianghai Chen ${ }^{1,+}$, Yong Zhou ${ }^{1,2,+}+\mathcal{D}$, Wei Lai ${ }^{3}$, Lifang $\mathrm{Hu}^{2,3}{ }^{-0}$, Lunwei Jiang ${ }^{1}$ and \\ Shiqiang Liu $1, *(\mathbb{D}$ \\ 1 Department of Biochemistry and Molecular Biology, College of Bioscience and Bioengineering, \\ Jiangxi Agricultural University, Nanchang 330045, China; 15870644120@163.com (L.C.); \\ yongzhou@jxau.edu.cn (Y.Z.); jlp_1999@163.com (L.J.) \\ 2 Key Laboratory of Crop Physiology, Ecology and Genetic Breeding, Ministry of Education, \\ Jiangxi Agricultural University, Nanchang 330045, China; lfhu_hn337@163.com \\ 3 College of Agronomy, Jiangxi Agricultural University, Nanchang 330045, China; 15797631915@163.com \\ * Correspondence: 1sq_hn306@163.com \\ + These authors contributed equally to this work.
}

Received: 30 December 2019; Accepted: 30 January 2020; Published: 5 February 2020

\begin{abstract}
The nuclear factor Y (NF-Y) transcription factors (TFs) play vital regulatory roles in diverse developmental processes and responses to abiotic stresses in plants. However, the NF- $Y$ genes remain largely unknown in cucumber. In this study, based on phylogenetic and protein structure

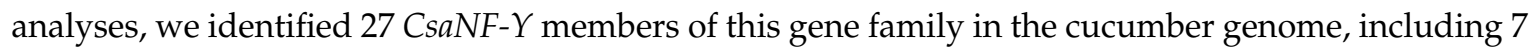
NF-YAs, 13 NF-YBs, and 7 NF-YCs. Their chromosome locations, gene structures, conserved domains, gene duplication, and promoter regions containing stress- and hormone-responsive cis-elements were also analyzed. As reported earlier, RNA-seq data showed that the expression of some CsaNF-Y genes was tissue-specific and varied during fruit development. The qRT-PCR results showed that all the detected CsaNF-Y genes were differentially regulated by drought and salt stress. Taken together, our findings provide a comprehensive understanding of CsaNF- $Y$ genes in the development and abiotic stress response of cucumber and lay the foundation for future crop improvement.
\end{abstract}

Keywords: cucumber; nuclear factor-Y (NF-Y); gene family; expression analysis; abiotic stress

\section{Introduction}

Nuclear factor Y (NF-Y) transcription factors (TFs), also known as heme activator protein (HAP) or CCAAT-binding factor $(\mathrm{CBF})$, are present in nearly all eukaryotes [1-3]. NF-Y TFs consist of three distinct subunits: NF-YA (HAP2 or CBF-B), NF-YB (HAP3 or CBF-A), and NF-YC (HAP5 or CBF-C), which are characterized by their conserved domains and sequence lengths $[4,5]$. Each of NF-Y TFs contain highly evolutionarily conserved domains in their central regions for DNA binding and NF-Y subunit interaction to form heterotrimeric complexes. In mammals and plants, NF-YB and NF-YC can form a dimer in the cytoplasm, and then this dimer is transferred into the nucleus to interact with NF-YA to form a NF-YA/NF-YB/NF-YC heterotrimer complex, which could bind to the CCAAT box in eukaryotic promoter regions to activate or inhibit the expression of downstream target genes [2,6,7].

Among yeast and mammals, each subunit of the NF-YA, NF-YB, and NF-YC proteins is encoded by a single gene, while each subunit of NF-Y protein is encoded by a multigene family in plants $[8,9]$, suggesting that all the three types of $N F-Y$ genes have undergone multiple duplications in plants. For example, 36 and 34 NF-Y genes were identified in Arabidopsis and rice (over 10 genes for each subunit), respectively $[10,11]$. In recent years, the $N F-Y$ gene family has been identified and characterized in 
a number of plant species, including 23 NF-Y genes in barley (Hordeum vulgare) [12], 24 in Citrus grandis [13], 24 in peach (Prunus persica) [14], 25 in castor bean (Ricinus communis) [15], 35 in tea plant (Camellia sinensis) [16], 42 in sorghum (Sorghum bicolor) [17], 44 in banana (Musa acuminata) [18], and 51 in cassava (Manihot esculenta) [19]. Many reports have shown that NF-Y TFs are involved in various physiological and biochemical processes of plants, such as flowering [20,21], seed germination [22], hypocotyl elongation [23], photomorphogenesis [24], root development [25,26], and grain filling and endosperm development [27-29]. In addition, the NF-Y TFs have been found to play critical roles in responses to a variety of biotic and abiotic stresses [2,5,19], and the biological functions of some NF-Y TFs in regulating plants' responses to various abiotic stresses have been studied in detail. For example, maize ZmNF-YA3 was found to have dual functions in photoperiod-dependent flowering and drought and high-temperature tolerance through binding to the promoter of FLOWERING LOCUS T-like12 (FT-like12) and interacting with several important TFs in JA- and ABA-associated signaling pathways [30]. A wheat nuclear factor Y (NF-Y) B subfamily gene TaNF-YB3; l can also confer tolerance to drought stress through the modulation of an ABA-signaling pathway [31].

Cucumber (Cucumis sativus L.) is one of the worldwide popular vegetable crops, and belongs to the genus Cucumis in Cucurbitaceae, which is prone to be threatened by various abiotic stresses [32]. Therefore, identification and characterization of genes related to stress resistance in cucumber would be of great significance to the improvement of resistance, yield, and quality of cucumber plants in future cucumber planting. In this study, we performed the genome-wide identification and systematic analysis of NF-Y TFs in cucumber, including genome distributions, gene and protein structures, phylogenetic relationships, and promoter sequences. Moreover, the expression patterns of the CsaNF- $Y$ genes in various tissues and fruit development stages, as well as in response to various abiotic stress treatments were also determined. The results will provide a basis for further revealing the biological roles of CsaNF-Y genes in the development and stress responses of cucumber.

\section{Materials and Methods}

\subsection{Plant Materials and Abiotic Stress Treatments}

Cucumber (Cucumis satious L. var. satious cv. 9930) was used in this study. Two-week-old cucumber seedlings were subjected to abiotic stress treatments including drought and salt as described in our previous study [32]. In brief, the seedlings were treated with 10\% PEG-6000 (Sigma-Aldrich, Shanghai, China) and $200 \mathrm{mM} \mathrm{NaCl}$ to induce drought stress and salt stress, respectively. The leaf samples were collected at different time points $(0,6,12$, and $24 \mathrm{~h})$, and each sample was harvested from over 10 plants with three biological replicates. All of the samples were immediately frozen in liquid nitrogen, and stored at $-80^{\circ} \mathrm{C}$ until use.

\subsection{Identification and Protein Property Analysis of NF-Y Members in Cucumber}

The Hidden Markov model (HMM) of the NF-Y conserved domains (PF02045 and PF00808) were downloaded from Pfam database (http://pfam.xfam.org/), and then used as Blast queries to search against the cucumber proteome (version 2.0, http:/cucurbitgenomics.org/organism/2) using HMMER software (http://hmmer.org) with an E-value of $1 \mathrm{e}^{-5}$. In addition, the amino acid sequences of the Arabidopsis NF-Y proteins were downloaded from TAIR (http://www.arabidopsis.org/) based on a previous report [10], and also used as query sequences for Blast searching against the cucumber proteome. After removal of the redundant sequences, the remaining sequences were checked by the Simple Modular Architecture Research Tool (SMART) (http://smart.embl-heidelberg.de/) to scan the complete NF-Y domains. The protein properties of NF-Y members, such as number of amino acids, molecular weight (MW), isoelectric points (pI), and grand average of hydropathicity (GRAVY), were examined with the ProtParam program (http: //web.expasy.org/protparam). 


\subsection{Chromosomal Location, Sequence Alignment, and Phylogenetic Analysis}

The chromosomal locations of the cucumber NF-Y genes were obtained in the cucumber genome database (http://cucurbitgenomics.org/organism/2), and drawn with the MapChart software (https:/www. wur.nl/en/show/Mapchart.htm) as previously described [33]. Duplication analysis was conducted using MCScanX software (http://chibba.pgml.uga.edu/mcscan2), and tandem and segmental duplication events were determined based on the previous description [34]. For sequence alignment of cucumber NF-Y proteins, their amino acid sequences were aligned using MAFFT (https://www.ebi.ac.uk/Tools/msa/mafft/) with default settings and visualized with GeneDoc. Moreover, multiple sequence alignments of NF-Y proteins from cucumber, Arabidopsis thaliana, and rice were also carried out with MAFFT, and a phylogenetic tree was constructed using the neighbor-joining (NJ) algorithm in MEGA 7.0 (https://www.megasoftware.net) with the bootstrap of 1000 replicates [35].

\subsection{Gene Structure and Promoter Region Analysis}

For gene structure analysis, the mRNA sequences and corresponding genomic DNA (gDNA) sequences of cucumber NF-Y genes were retrieved from the cucumber genome database, and the gene structures were determined by comparing the mRNA and gDNA sequences using GSDS tool (http://gsds.cbi.pku.edu.cn/). For promoter region analysis, the cis-elements in the $2.0 \mathrm{~kb}$ upstream region from translation start code ATG of each cucumber NF-Y gene were investigated using the PlantCARE tool (http://bioinformatics.psb.ugent.be/webtools/plantcare/html/).

\subsection{Expression Analysis of Cucumber NF-Y Genes With RNA-seq Data}

For analysis of the cucumber NF-Y genes in different tissues, the RNA-seq data from ten different tissues (unexpanded ovary, fertilized ovary, unfertilized ovary, root, stem, leaf, male flower, female flower, tendril, and basal tendril) were retrieved in Sequence Read Archive under BioProject PRJNA80169 [36]. The expression of the cucumber NF-Y genes was analyzed based on these data, and the expression values were indicated as the RPKM values. To analyze the cucumber NF- $Y$ genes during fruit ripening, the RPKM values of each NF-Y gene in leaves and fruits (10 days after pollination, $10 \mathrm{DAP} ; 20 \mathrm{DAP} ; 30 \mathrm{DAP}$; and $40 \mathrm{DAP}$ ) were obtained in the fruitENCODE database (http://www.epigenome.cuhk.edu.hk/encode.html). The expression values were log2-transformed and the heat maps were created with the TBtools software [37].

\subsection{RNA Extraction and Quantitative RT-PCR ( $q R T-P C R)$}

Total RNA was isolated using the Eastep Super Total RNA Extraction Kit (Promega, Madison, WA, USA) according to the manufacturer's protocols. The genomic DNA contamination was eliminated by RNase-free DNase I (TianGen, Beijing, China), and the first-strand cDNA was obtained using the M-MLV reverse transcriptase (Invitrogen, Carlsbad, CA, USA). The qRT-PCR was performed using the TB Green Premix Ex TaqII Kit (TaKaRa Biotechnology, Dalian, China) and the amplification program was set as previously described [32]. Three biological replicates were performed to test the expression of all the selected genes. Their relative transcript levels were calculated with the $2^{-\Delta \Delta C T}$ method by using CsACT3 as an internal reference [38]. The qRT-PCR data were analyzed with one-way analysis of variance using Tukey's "Honest Significant Difference" method, and the significance was set at $P<0.05$. Gene-specific primers used for qRT-PCR are presented in Table S1.

\section{Results}

\subsection{Genome-Wide Identification of the NF-Y Gene Family in Cucumber}

After the removal of redundant sequences and conserved domain analysis, $27 \mathrm{NF-} Y$ genes were identified in the cucumber genome, including 7 NF-YAs, 13 NF-YBs, and 7 NF-YCs, which were named in accordance with their positions and relative distance on cucumber chromosomes (Table 1). 
The gDNA and CDS lengths of the identified CsaNF-Y genes ranged from $357 \mathrm{bp}$ (CsaNF-YB3) to $5290 \mathrm{bp}$ (CsaNF-YA4), and $357 \mathrm{bp}$ (CsaNF-YB3) to $1035 \mathrm{bp}$ (CsaNF-YA4), respectively. Besides, these genes encoded proteins ranging from 118 (CsaNF-YB3) to 344 (CsaNF-YA4) amino acids in length, and the MWs and pIs values of CsaNF-Y proteins varied from 13.09 to $38.98 \mathrm{kDa}$ and 4.68 to 9.53 , respectively (Table 1). The grand average of hydropathicity (GRAVY) values of the CsaNF-Y proteins were calculated to be lower than 0 except for CsaNF-YC2 (Table 1), suggesting that they are hydrophilic.

\subsection{Phylogenetic Analysis, Chromosomal Location, and Gene Duplication of the NF-Y Gene Family}

To further understand the phylogenetic relationships of NF-Y proteins between cucumber and other plant species, a phylogenetic tree was generated with the NF-Y amino acid sequences from cucumber, Arabidopsis [10,39], and rice [11]. As shown in the phylogenetic tree (Figure 1), these NF-Y proteins could be classified into three subfamilies named as NF-YA, NF-YB, and NF-YC, which was in accordance with our identification results. In addition, cucumber NF-Ys displayed closer relationships with AtNF-Ys than with OsNF-Ys (Figure 1).

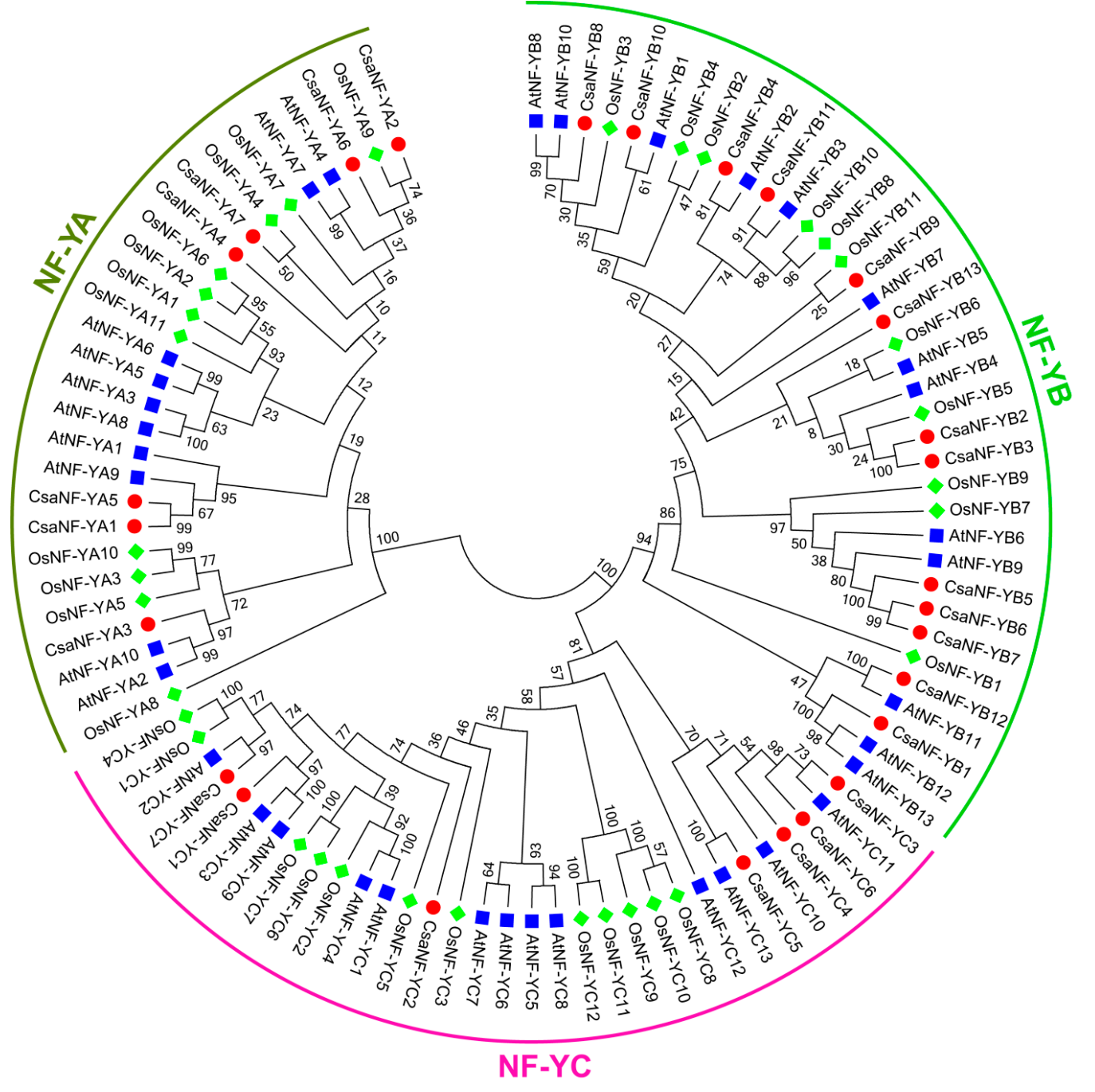

Figure 1. Phylogenetic analysis of NF-Y gene family among cucumber, Arabidopsis, and rice. Full-length NF-Y proteins from cucumber, Arabidopsis, and rice were aligned using MAFFT, and the phylogenetic tree was created with MEGA 7.0 by the neighbor joining (NJ) method with 1000 bootstrap replicates. The protein IDs from Arabidopsis and rice are listed in Table S2. 
Table 1. Identification and characterization of nuclear factor $Y(N F-Y)$ family genes in cucumber.

\begin{tabular}{|c|c|c|c|c|c|c|c|c|}
\hline \multirow{2}{*}{ Nomenclature } & \multirow{2}{*}{ Locus } & \multirow{2}{*}{ Chromosomal Position } & \multirow{2}{*}{ gDNA (bp) } & \multirow{2}{*}{ CDS (bp) } & \multicolumn{4}{|c|}{ Protein } \\
\hline & & & & & Length (aa) & MW (KDa) & pI & GRAVY \\
\hline CsaNF-YA1 & Csa1G613580 & Chr1: 24112521-24117512 & 3199 & 810 & 269 & 29.52 & 6.56 & -0.984 \\
\hline CsaNF-YA2 & Csa3G644810 & Chr3: 25263223-25264735 & 1513 & 444 & 147 & 16.91 & 9.00 & -0.122 \\
\hline CsaNF-YA3 & Csa3G782710 & Chr3: 30348958-30352913 & 3395 & 975 & 324 & 35.46 & 9.40 & -0.436 \\
\hline CsaNF-YA4 & Csa4G159320 & Chr4: 8340821-8346110 & 5290 & 1035 & 344 & 38.98 & 8.63 & -0.489 \\
\hline CsaNF-YA5 & Csa6G439960 & Chr6: 20632195-20637169 & 3212 & 1026 & 341 & 37.10 & 6.53 & -0.895 \\
\hline CsaNF-YA6 & Csa6G525440 & Chr6: 28505741-28509554 & 3814 & 609 & 202 & 22.53 & 8.58 & -1.032 \\
\hline CsaNF-YA7 & Csa7G428130 & Chr7: 16427189-16430567 & 2325 & 957 & 318 & 35.57 & 6.96 & -0.733 \\
\hline CsaNF-YB1 & Csa1G015800 & Chr1: 2159806-2163445 & 3332 & 471 & 156 & 17.47 & 4.68 & -0.547 \\
\hline CsaNF-YB2 & Csa1G569510 & Chr1: 21018573-21018944 & 372 & 372 & 123 & 14.11 & 6.10 & -0.903 \\
\hline CsaNF-YB3 & Csa1G569530 & Chr1: 21023622-21023978 & 357 & 357 & 118 & 13.63 & 5.29 & -0.815 \\
\hline CsaNF-YB4 & Csa3G047800 & Chr3: 3332696-3333441 & 555 & 555 & 184 & 19.54 & 5.46 & -0.630 \\
\hline CsaNF-YB5 & Csa3G048940 & Chr3: $3452386-3453255$ & 870 & 411 & 136 & 15.10 & 5.51 & -0.680 \\
\hline CsaNF-YB6 & Csa3G049440 & Chr3: $3458401-3459120$ & 720 & 720 & 239 & 26.50 & 5.29 & -0.495 \\
\hline CsaNF-YB7 & Csa3G055940 & Chr3: 3469618-3470370 & 753 & 666 & 221 & 24.78 & 5.04 & -0.533 \\
\hline CsaNF-YB8 & Csa3G736780 & Chr3: 28408903-28412596 & 3242 & 522 & 173 & 18.76 & 6.09 & -0.758 \\
\hline CsaNF-YB9 & Csa4G037610 & Chr4: 3160771-3161568 & 722 & 678 & 225 & 24.73 & 7.76 & -0.638 \\
\hline CsaNF-YB10 & Csa5G175930 & Chr5: 7498740-7502620 & 3881 & 528 & 175 & 19.17 & 5.26 & -0.709 \\
\hline CsaNF-YB11 & Csa6G112490 & Chr6: 7775649-7777337 & 925 & 606 & 201 & 20.62 & 6.31 & -0.636 \\
\hline CsaNF-YB12 & Csa6G425700 & Chr6: 19919807-19922480 & 2190 & 483 & 160 & 18.05 & 4.77 & -1.087 \\
\hline CsaNF-YB13 & Csa7G395250 & Chr7: 15134980-15135499 & 520 & 483 & 160 & 17.37 & 5.03 & -0.781 \\
\hline CsaNF-YC1 & Csa1G570150 & Chr1: 21086208-21089499 & 1214 & 780 & 259 & 28.70 & 5.89 & -0.518 \\
\hline CsaNF-YC2 & Csa4G049050 & Chr4: 3878004-3878711 & 513 & 360 & 119 & 13.09 & 7.76 & 0.035 \\
\hline CsaNF-YC3 & Csa5G583350 & Chr5: 20754210-20758105 & 3896 & 852 & 283 & 31.66 & 5.14 & -0.918 \\
\hline CsaNF-YC4 & Csa6G294440 & Chr6: $14150180-14153107$ & 1843 & 573 & 190 & 21.31 & 9.53 & -1.115 \\
\hline CsaNF-YC5 & Csa6G365140 & Chr6: 16501105-16501524 & 420 & 420 & 139 & 15.72 & 9.01 & -0.658 \\
\hline CsaNF-YC6 & Csa6G426970 & Chr6: 20154306-20158203 & 3898 & 843 & 280 & 31.23 & 4.90 & -0.816 \\
\hline CsaNF-YC7 & Csa7G051420 & Chr7: 3270343-3272852 & 2510 & 801 & 266 & 29.96 & 5.96 & -0.705 \\
\hline
\end{tabular}


All the CsaNF-Y genes were distributed throughout all seven chromosomes of cucumber, except for chromosome 2 (Figure 2). Chromosomes 3 and 6 harbored the largest number of CsaNF-Y genes (seven genes each), followed by chromosome 1 (five genes), and two chromosomes (chromosomes 4 and 7) each contained three CsaNF-Y genes, while chromosome 5 harbored the fewest CsaNF-Y genes (two genes) (Figure 2).

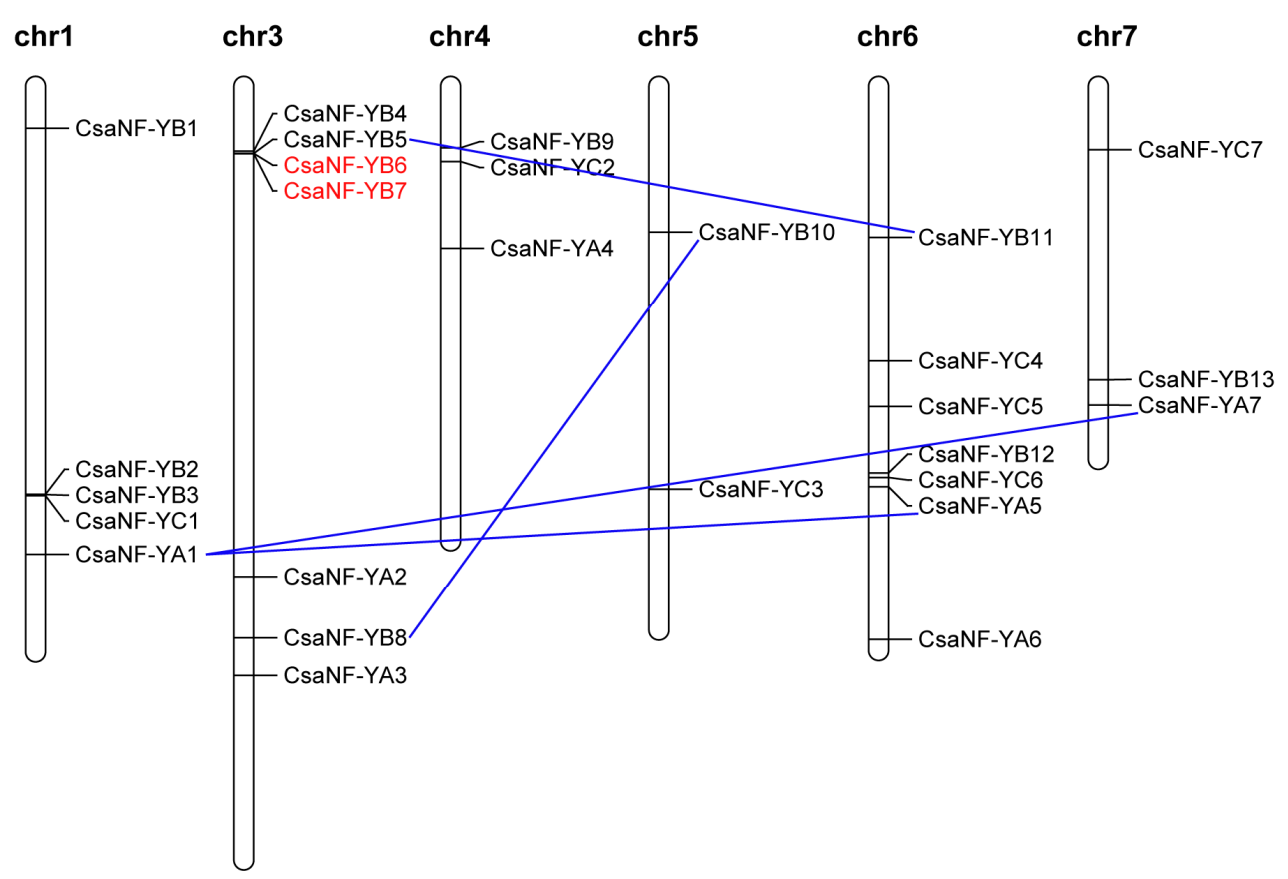

Figure 2. Chromosomal distribution of cucumber $N F-Y$ genes. The chromosome number is indicated at the top, and chromosome 2 harbors no CsaNF-Y genes.

To identify potential segmental and tandem duplications within the cucumber genome, we assessed the duplication events among the identified CsaNF-Y genes using the criteria from a previous study [34]. Tandem duplication was detected for one pair of CsaNF-Y genes, CsaNF-YB6 and CsaNF-YB7, whereas segmental duplication was found for four pairs of genes, including CsaNF-YA1 and CsaNF-YA5, CsaNF-YA1 and CsaNF-YA7, CsaNF-YB5 and CsaNF-YB11, and CsaNF-YB8 and CsaNF-YB10 (Figure 2).

\subsection{Conserved Domain Analysis of CsaNF-Y Proteins}

Full-length CsaNF-YAs, CsaNF-YBs, and CsaNF-YCs were aligned by MAFFT to determine the evolutionarily conserved domains for DNA-binding and NF-Y subunit interaction. The multiple alignment results revealed that a heterodimerization domain (for interaction with other subunit of NF-Y) and a DNA-binding domain (for recognition of CCAAT binding sites) were commonly found in CsaNF-Y proteins, and these domains of CsaNF-YAs were found to be much more conserved than those of CsaNF-YBs and CsaNF-YCs (Figure 3). The CsaNF-YA proteins contained two highly conserved sub-domains, one domain for interaction with NF-YB/C and one domain for DNA binding, which were separated by a relatively conserved linker (Figure 3A). In addition, three histidine $(\mathrm{H})$ and three arginine (R) residues were present in DNA binding domain of the majority of the CsaNF-YAs, while CsaNF-YA2 had obviously different residues from other six CsaNF-YA members in DNA binding domain (Figure 3A). Furthermore, the core histone structures including the HFM (histone fold motif) of the core histone H2B and H2A were present in CsaNF-YB and CsaNF-YC proteins, respectively (Figure 3B,C). CsaNF-YB proteins contained a DNA-binding domain, a NF-YA interaction domain, and a NF-YC interaction domain, and the region of the NF-YC interaction domain was so long that it overlapped with the NF-YA interaction and DNA-binding domains (Figure 3B). Most CsaNF-YC proteins contained one DNA-binding domain, two NF-YA interaction domains and one 
NF-YB interaction domain. The two NF-YA interaction domains were separated by the NF-YB interaction domain, and the DNA-binding domain was embedded in the first NF-YA interaction domain (Figure 3C).

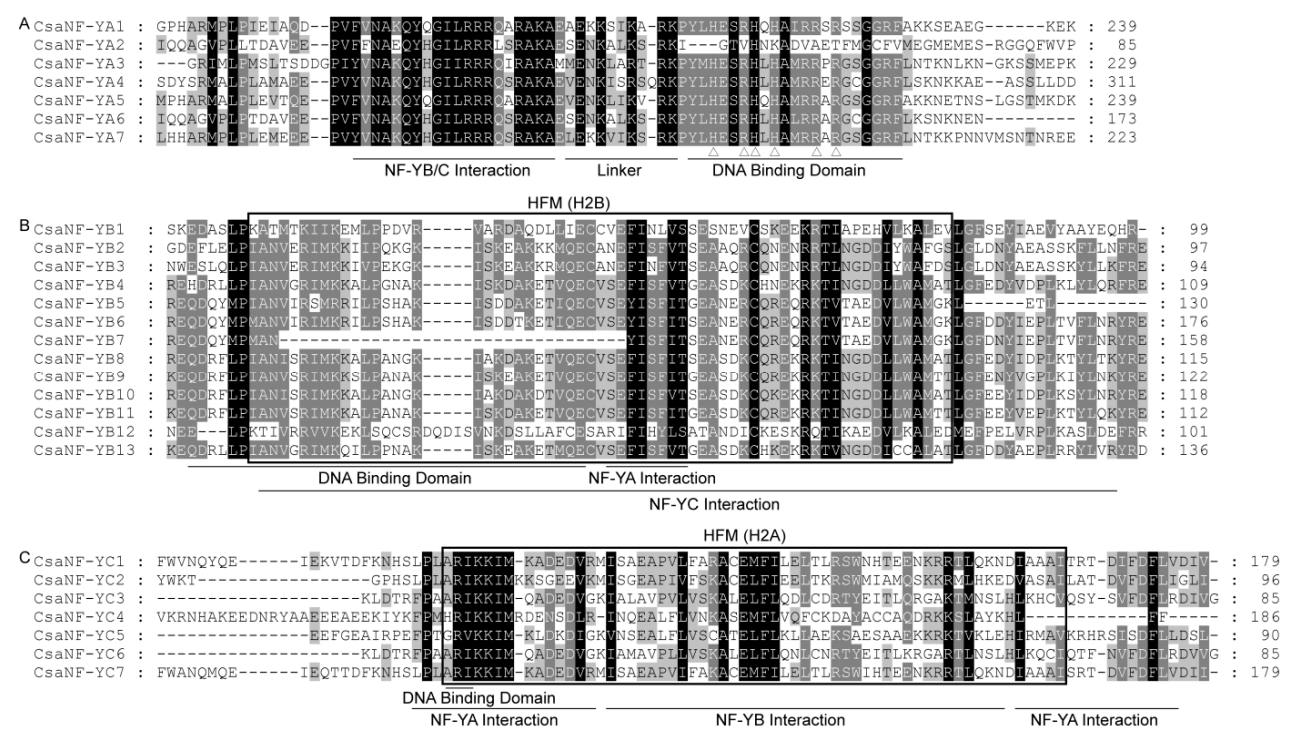

Figure 3. Multiple alignments of CsaNF-Y protein sequences. (A) Multiple alignments of CsaNF-YA protein sequences. (B) Multiple alignments of CsaNF-YB protein sequences. (C) Multiple alignments of CsaNF-YC protein sequences. The evolutionarily conserved domains including the DNA-binding domains and the NF-Y subunit interaction domains are underlined. The histone-fold motif (HFM) of the core histones (H2A and H2B) are boxed. Three histidine $(\mathrm{H})$ and three arginine $(\mathrm{R})$ residues in the DNA binding domain of the CsaNF-YA proteins are marked with triangles.

\subsection{Gene Structure Analysis of CsaNF-Y Genes}

To determine the exon-intron structures of the CsaNF- $Y$ genes, we compared the mRNA sequences with the corresponding gDNA sequences by GSDS tool. As a result, the intron numbers of CsaNF-Y genes varied from 0 to 5 (Figure 4). Most CsaNF-YA genes contained four introns, with the exception of CsaNF-YA2 and CsaNF-YA4, which had two and five introns, respectively. Among the CsaNF-YB genes, three contained four introns (CsaNF-YB1, CsaNF-YB8, and CsaNF-YB10), one contained two introns (CsaNF-YB12), two contained only one intron (CsaNF-YB5 and CsaNF-YB7), while seven other $C s a N F-Y B$ genes were intronless (Figure 4). As for the CsaNF-YC genes, the largest number of introns was found for CsaNF-YC3 and CsaNF-YC6 (five introns); CsaNF-YC7 and CsaNF-YC4 contained one and two introns, respectively; while CsaNF-YC1, CsaNF-YC2, and CsaNF-YC5 had no intron (Figure 4).

\subsection{Bioinformatics Analysis of Putative CsaNF-Y Promoters}

To investigate the potential functions of CsaNF- $Y$ genes, 2.0-kb promoter region of each CsaNF- $Y$ gene was analyzed by PlantCARE. A total of 15 cis-elements were found in the promoters of the CsaNF- $Y$ genes, including six stress-responsive elements and nine hormone-responsive elements (Figure S1). Amongst these cis-elements, ARE (anaerobic induction element) and ERE (ethylene-responsive element) were the most abundant stress-responsive element and hormone-responsive element, being present in 24 and 21 CsaNF-Y genes, respectively. In addition, five other stress-responsive elements, including MBS, LTR, W-box, WUN-motif, and TC-rich repeats, were also present in a series of CsaNF-Y genes (Figure S1), indicating their important roles in responses to various stresses. Besides ERE, five kinds of other hormone-responsive cis-elements, including abscisic acid (ABA)-responsive element (ABRE), methyl jasmonate (MeJA)-responsive element (CGTCA-motif), salicylic acid (SA)-responsive elements (TCA-element), auxin-responsive elements (AuxRR-core and TGA-element), and gibberellin-responsive elements (P-box, GARE-motif, and TATC-box), were found in the promoter regions of 16, 12, 11, 12, and 
14 CsaNF-Y genes, respectively (Figure S1), indicating that the CsaNF-Y genes may also play important roles in hormone responses.

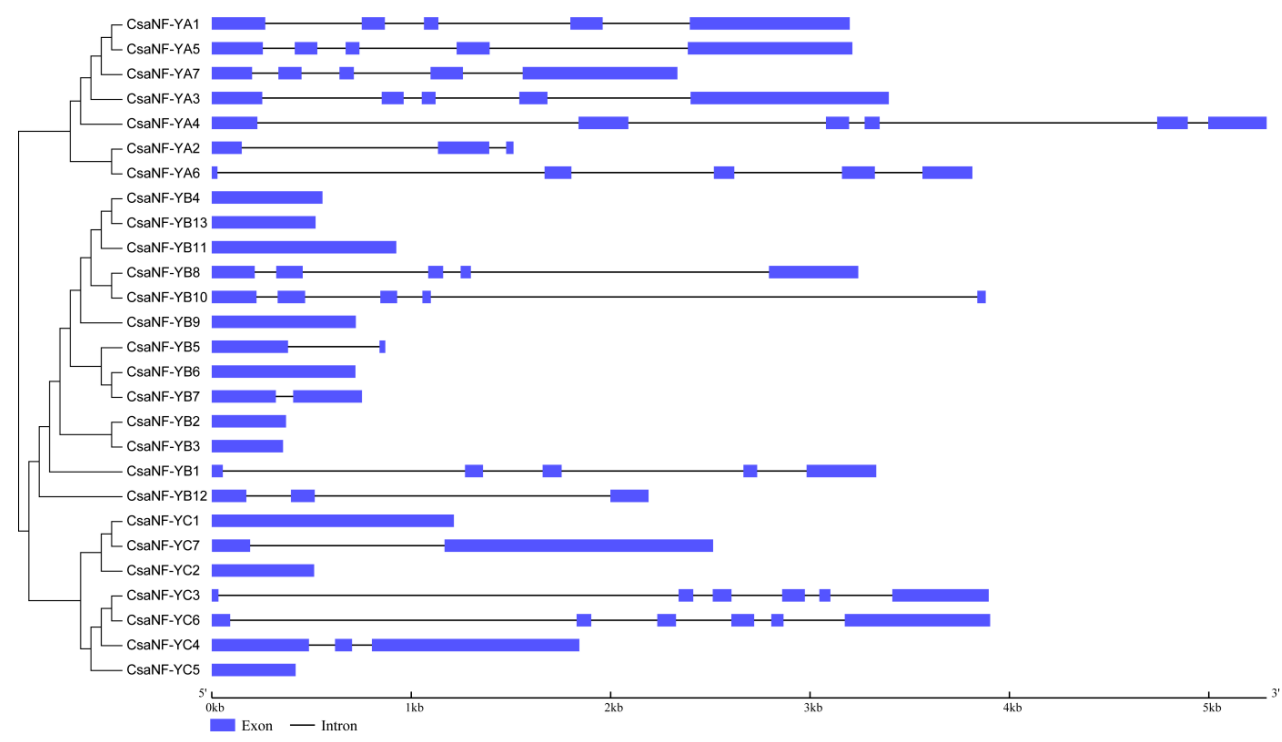

Figure 4. Gene structure analysis of CsaNF-Y genes according to the phylogenetic relationship. The blue boxes and black lines indicate exons and introns, respectively, and their lengths can be estimated by the scale bar at the bottom.

\subsection{Expression Profiles of CsaNF-Y Genes in Various Tissues and Organs}

To gain insights into the tissue expression profiles of CsaNF- $Y$ genes, we analyzed their expression levels in ten tissues (unexpanded ovary, fertilized ovary, unfertilized ovary, root, stem, leaf, male flower, female flower, tendril, and basal tendril) using publicly available RNA-seq data [36]. According to the transcriptomic dataset, five genes (CsaNF-YB2, CsaNF-YB3, CsaNF-YB5, CsaNF-YB6, and CsaNF-YB7) showed RPKM values lower than 1.0 in all the tested tissues, while other $22 C s a N F-Y$ genes were expressed in at least one of the tested tissues (Figure S2). Several genes, such as CsaNF-YB1, CsaNF-YB8, CsaNF-YC1, and CsaNF-YC7, had relatively higher expression levels in all detected tissues (Figure S2), suggesting that they might play essential roles in plant growth. In addition, some CsaNF- $Y$ genes exhibited tissue-specific expression patterns, suggesting that these genes possibly play certain roles in organ development of cucumber. For example, CsaNF-YA3 and CsaNF-YB13 were more abundantly expressed in roots, and CsaNF-YB1 and CsaNF-YC7 had higher expression in unfertilized ovaries. In addition, CsaNF-YB9 and CsaNF-YC2 were highly expressed in male and female flowers, respectively (Figure S2).

To better examine the functions of CsaNF-Y genes in the growth and development of cucumber, their expression profiles during fruit development were analyzed according to a previous study [40]. The RPKM values of 10 CsaNF-Y genes (CsaNF-YA2, CsaNF-YA4, CsaNF-YB2, CsaNF-YB3, CsaNF-YB5, CsaNF-YB6, CsaNF-YB7, CsaNF-YB13, CsaNF-YC2, and CsaNF-YC6) were 0 or lower than 1.0 during fruit development, suggesting that they are not expressed in fruit (Figure S3). The expression levels of several CsaNF-Y genes, such as CsNF-YA1, CsNF-YA3, CsNF-YA6, and CsNF-YC7, gradually increased during fruit development and reached the maximum at $40 \mathrm{DPA}$, indicating their vital roles in fruit ripening. CsNF-YA5 and CsNF-YA7 exhibited up-regulated expression at the onset of fruit development (20 DPA or 30 DPA), but their expression levels were significantly down-regulated at 40 DPA (Figure S3). In contrast, CsaNF-YB4, CsaNF-YC4 and CsaNF-YC5 showed significant decreases in expression during fruit development (Figure S3), suggesting their possible roles in suppressing the ripening of fruit. 


\subsection{Expression Patterns of CsaNF-Y Genes in Response To Various Abiotic Stresses}

To study the roles of CsaNF-Y genes in abiotic stress response, the expression profiles of six selected CsaNF-Y genes (2 each in distinct subunits) in response to drought and salt stresses were assessed using qRT-PCR. All the six CsaNF- $Y$ genes displayed relatively high expression in various tissues and organs (Figures S2 and S3). Upon the drought stress, the expression of four CsaNF-Y genes (CsaNF-YA5, CsaNF-YA6, CsaNF-YB4, and CsaNF-YC7) increased to varying degrees, while that of $C s a N F-Y C 3$ decreased (Figure 5). The expression levels of the up-regulated CsaNF- $Y$ genes peaked at $12 \mathrm{~h}$, with the exception of CsaNF-YB4, whose expression reached the peak at $6 \mathrm{~h}$ (Figure 5). Under salt stress conditions, all of the tested CsaNF-Y genes were significantly up-regulated (Figure 6). The expression levels of CsaNF-YA5 and CsaNF-YB11 significantly increased and reached the highest values at $6 \mathrm{~h}$, while those of $C s a N F-Y B 4, C s a N F-Y C 3$, and CsaNF-YC7 reached the maximum values until $24 \mathrm{~h}$ (Figure 6). Notably, CsaNF-YA6 was the most observably induced gene, and its expression sharply increased at $12 \mathrm{~h}$ and displayed a 23.2-fold change under salt stress (Figure 6).
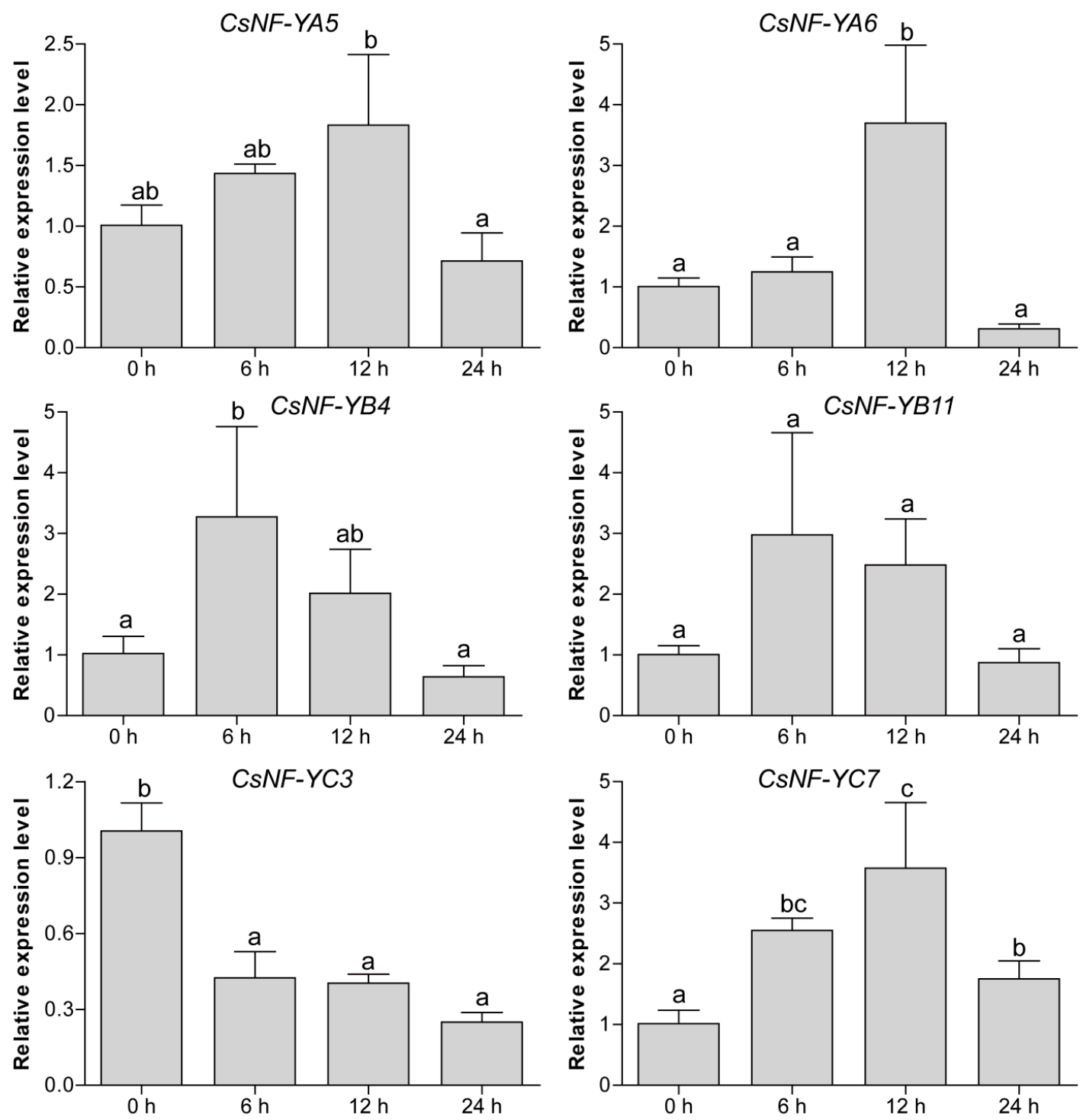

Figure 5. Expression analysis of six selected CsaNF-Y genes in response to drought stress using qRT-PCR. Vertical bars indicate the standard error of the mean, and different letters are significantly different. 

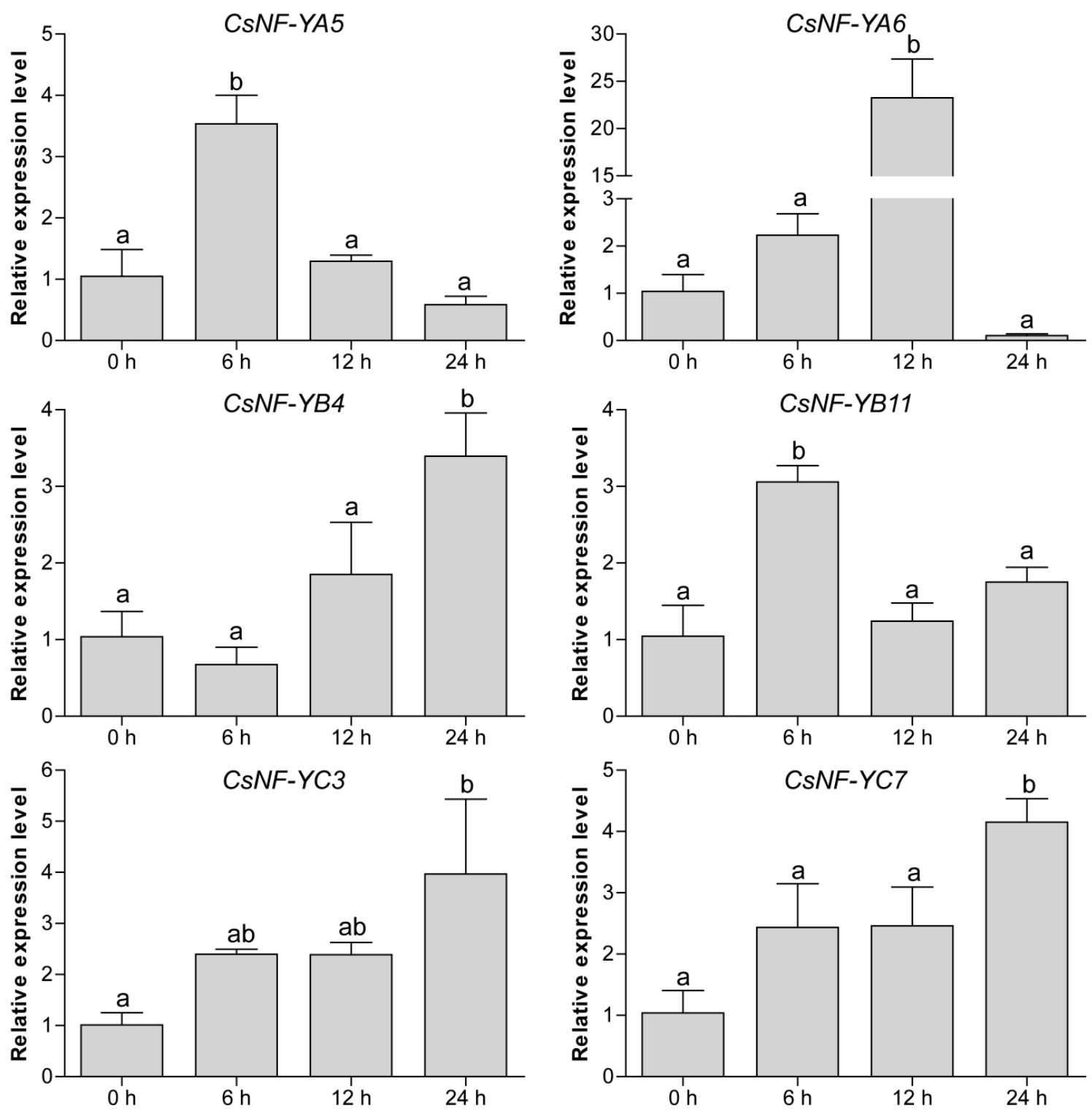

Figure 6. qRT-PCR expression analysis of six selected CsaNF-Y genes in response to salt stress. Vertical bars indicate the standard error of the mean, and different letters are significantly different.

\section{Discussion}

In this study, genome-wide identification and characterization of $N F-Y$ gene family were carried out, and a total of 27 CsaNF-Y genes (including 7 NF-YAs, 13 NF-YBs, and 7 NF-YCs) were identified in cucumber (Table 1). Similar to that in other plants, the NF-Y subunit in cucumber also exists in multiple copies (Table 1; Figure 1), suggesting that the cucumber NF-Y gene family has undergone a number of duplication events. In the present study, eight segmentally duplicated genes and two tandemly duplicated genes were identified in the cucumber genome (Figure 2). In previous research, a total of 15 and 4 OsNF- $Y$ genes could be assigned as segmentally and tandemly duplicated genes in rice [11], 12 and 9 SiNF- $Y$ genes made up to six segmental duplication events and four tandem duplication events, respectively [41]. These results indicate that both of segmental and tandem duplications play a crucial role in the expansion of NF-Y genes across different plants.

The phylogenetic analysis suggested that the NF-Y proteins from cucumber, Arabidopsis, and rice could be classified into three subfamilies named as NF-YA, NF-YB, and NF-YC, and NF-Y proteins from cucumber had closer phylogenetic relationship with those from Arabidopsis than with 
those from rice (Figure 1). In addition, the multiple sequence alignments revealed that nearly all CsaNF-Y proteins possess evolutionarily conserved domains for DNA binding and heterotrimerization (Figure 3), which is in agreement with the NF-Y protein characteristics [14,16,41,42]. Previous studies have shown that NF-YA genes always display a highly structured intron-exon organization and the majority of them have 3-6 introns, while the NF-YB and NF-YC genes exhibit variable intron/exon organizations [15,16,41]. In this study, $C s a N F-Y A$ genes contained 2-5 introns, and multiple intron/exon organizations were observed for $N F-Y B$ and NF-YC genes (Figure 4), which is in agreement with previous studies, revealing the conserved features of the evolution of these subunits in plants. In addition, the CsaNF-YBs (7/13) and CsaNF-YCs (3/7) were prevalently lack of introns (Figure 4), which is a universal feature of $N F-Y B$ and NF-YC genes in other plant species, such as peach, in which 6 out of $12 N F-Y B s$ and 4 out of 6 NF-YCs have no introns [14]; tea plant, in which 7 out of 15 NF-YBs and 6 out of $10 \mathrm{NF-YCS}$ have no introns [16]; castor bean, in which 8 out of $12 \mathrm{NF-YBs}$ and 3 out of 7 NF-YCs have no introns [15]; chickpea, in which 11 out of 21 NF-YBs and 7 out of 11 NF-YCs have no introns [43]; and banana, in which 12 out of 16 NF-YBs and 12 out of 14 NF-YCS have no introns [18]. The similar conserved domain arrangements and intron/exon organizations in each subunit indicate that NF-Y genes are highly conserved during the evolution of plants, which may contribute to their crucial biological functions.

It has been demonstrated that NF-Y genes have diverse expression patterns in different tissues of plants. For example, five RcNF-Y genes were highly and specifically expressed in the early and later stages of developing endosperm in castor bean [15]. In walnut, 24 out of $33 \mathrm{JrNF}-\mathrm{Y}$ genes displayed higher expression levels in female flower buds than in leaf buds, suggesting that these genes play direct or indirect roles in the process of flower bud development [42]. In this study, nearly all CsaNF-YA genes were highly expressed in ovaries at different stages (Figure S2), indicating that they might participate in the regulation of ovary development in cucumber. In addition, some CsaNF- $Y$ genes showed high abundance of expression in flowers (Figure S2), suggesting their importance in the control of flowering. Amongst them, CsaNF-YB4 and CsaNF-YB11 are orthologous to AtNF-YB2 and AtNF-YB3, both of which have been identified as regulators that control photoperiod-dependent flowering time by interacting with other AtNF-Y subunit proteins [44]. In addition, OsNF-YB8 and OsNF-YB10 were clustered with CsaNF-YB4, CsaNF-YB11, AtNF-YB2, and AtNF-YB3 (Figure 1), and overexpression of OsNF-YB8 and OsNF-YB10 could complement the late flowering phenotype of Arabidopsis $n f-y b 2 n f-y b 3$ mutants [21]. CsaNF-YC1 is orthologous to AtNF-YC3 and AtNF-YC9, which were found to have overlapping functions in CONSTANS-mediated floral promotion [24,45]. It should be noted that CsaNF-YB13 was specifically expressed in root (Figure S2), indicating its particular role in root development. Furthermore, the expression levels of several CsaNF-Y genes, such as CsNF-YA1, CsNF-YA3, CsNF-YA6, and CsNF-YC7, gradually increased throughout the fruit development stage, while some other CsaNF-Y genes, such as CsaNF-YB4, CsaNF-YB11, CsaNF-YC4, and CsaNF-YC5, showed significant decreases in expression during fruit development (Figure S3), suggesting that they might participate in the regulation of fruit development, and have the potential to improve the fruit yield of cucumber.

Recently, numerous reports have revealed the important functions of NF- $Y$ genes in mediating abiotic stress tolerance in plants [2,46]. In this study, six stress-responsive elements and nine hormone-responsive elements were observed in the promoters of CsaNF-Y genes (Figure S1), suggesting that some of the CsaNF-Y genes may also participate in stress responses. We then determined the expression of NF- $Y$ genes under drought and salt stresses by qRT-PCR. Under the two stress conditions, four CsaNF-Y genes (CsaNF-YA5, CsaNF-YA6, CsaNF-YB4, and CsaNF-YC7) were up-regulated at certain time points, suggesting that these genes may play positive roles in response to these abiotic stresses (Figures 5 and 6). The possible roles of these CsaNF-Y genes in regulating stress tolerance should be further determined through genetic transformation. In a previous study, Picea wilsonii PwNF-YB3 transcript was induced by salinity and PEG treatments, and overexpression of PwNF-YB3 resulted in enhanced drought and salt tolerance in transgenic Arabidopsis plants [47]. Similarly, bermudagrass 
CdtNF-YC1 was up-regulated under salt, drought, and ABA stresses, and its overexpression conferred tolerance to drought and salt stress in transgenic rice and seashore paspalum plants [48,49]. Therefore, the four CsaNF-Y genes (CsaNF-YA5, CsaNF-YA6, CsaNF-YB4, and CsaNF-YC7) may have the potential roles in the resistance to drought and salt stress. In addition, CsaNF-YB11 was induced by salt stress but not by drought stress (Figures 5 and 6), implying its special role in response to salt stress. CsaNF-YB4 and $C s a N F-Y B 11$ are homologous with $A t N F-Y B 2$ and $A t N F-Y B 3$ (Figure 1), which were reported to specifically enhance drought and heat stress tolerance in Arabidopsis through specifically activating the drought-inducible and heat-inducible genes, respectively [50]. Moreover, CsaNF-YC3 was induced by salt stress but suppressed by drought stress (Figures 5 and 6), indicating that it may have opposite functions in response to salt and drought stress. In a previous study, overexpression of TaNF-YA10-1 in Arabidopsis significantly increased the sensitivity of the plant to salinity, but the transgenic plants displayed enhanced drought tolerance [51]. Therefore, the CsaNF-Ys are crucial candidate genes for genetic improvement of drought and salt stress tolerance in cucumber.

\section{Conclusions}

In this study, a total of 27 NF-Y family genes including 7 NF-YAs, 13 NF-YBs, and 7 NF-YCs were identified in the whole genome of cucumber. A comprehensive analysis of these NF- $Y$ genes was preformed, including phylogenetic relationships, genomic organizations, gene duplication events, conserved domains, gene structures, and promoter regions. In addition, RNA-seq and qRT-PCR assays were employed to investigate the possible roles of $C s a N F-Y$ genes in fruit development and abiotic stress response. The in silico expression results highlighted the importance of CsaNF- $Y$ genes in fruit development, and modulation of the expression levels of CsaNF-Y genes in response to drought and salt stresses indicates their key roles in stress tolerance. Our results provide the relevant information for further investigation of the biological functions of $N F-Y$ genes and improving cucumber agricultural traits in the future.

Supplementary Materials: The following are available online at http://www.mdpi.com/2073-4395/10/2/236/s1: Table S1: The gene-specific primers used for qRT-PCR, Table S2: NF-Y IDs from Arabidopsis and rice, Figure S1: Analysis of stress-responsive and hormone-responsive cis-elements in $C s a N F-Y$ genes. The numbers of cis-elements in the promoter region of each CsaNF- $Y$ gene are boxed and colored, Figure S2: Expression profiles of CsaNF-Y genes in various cucumber tissues and organs. The vertical color scale from red to blue indicates the expression levels of CsaNF-Y genes in log2-transformed RPKM + 1 values, Figure S3: Expression profiles of CsaNF- $Y$ genes during fruit development. The vertical color scale from red to blue indicates the expression levels of CsaNF-Y genes in log2-transformed RPKM +1 values.

Author Contributions: Data curation, L.C., Y.Z., W.L. and S.L.; funding acquisition, S.L.; investigation, Y.Z., L.H. and L.J.; methodology, L.C., Y.Z., W.L., L.H. and L.J.; resources, L.J. and S.L.; software, L.C., Y.Z., W.L., L.H. and S.L.; validation, L.C., Y.Z. and W.L.; visualization, L.H.; writing-original draft, L.C., Y.Z. and S.L.; writing-review \& editing, S.L. All authors have read and agreed to the published version of the manuscript.

Funding: This work was funded by the Key Project of Youth Science Foundation of Jiangxi Province (20171ACB21025 and 20181ACB20012), and the National Natural Science Foundation of China (31660578).

Conflicts of Interest: The authors declare that they have no conflict of interest.

\section{References}

1. Laloum, T.; De Mita, S.; Gamas, P.; Baudin, M.; Niebel, A. CCAAT-box binding transcription factors in plants: Y so many? Trends Plant Sci. 2013, 18, 157-166. [CrossRef] [PubMed]

2. Myers, Z.A.; Holt, B.F. NUCLEAR FACTOR-Y: Still complex after all these years? Curr. Opin. Plant. Biol. 2018, 45, 96-102. [CrossRef]

3. Dolfini, D.; Zambelli, F.; Pavesi, G.; Mantovani, R. A perspective of promoter architecture from the CCAAT box. Cell Cycle 2009, 8, 4127-4137. [CrossRef] [PubMed]

4. Petroni, K.; Kumimoto, R.W.; Gnesutta, N.; Calvenzani, V.; Fornari, M.; Tonelli, C.; Holt, B.F.; Mantovani, R. The promiscuous life of plant NUCLEAR FACTOR Y transcription factors. Plant. Cell 2012, 24, 4777-4792. [CrossRef] [PubMed] 
5. Zanetti, M.E.; Ripodas, C.; Niebel, A. Plant NF-Y transcription factors: Key players in plant-microbe interactions, root development and adaptation to stress. Biochim. Biophys. Acta. Gene Regul. Mech. 2017, 1860, 645-654. [CrossRef] [PubMed]

6. Li, S.; Li, K.; Ju, Z.; Cao, D.; Fu, D.; Zhu, H.; Zhu, B.; Luo, Y. Genome-wide analysis of tomato NF-Y factors and their role in fruit ripening. Can. J. Plant Sci 2016, 17, 36. [CrossRef]

7. Dolfini, D.; Gatta, R.; Mantovani, R. NF-Y and the transcriptional activation of CCAAT promoters. Crit. Rev. Biochem. Mol. Biol. 2012, 47, 29-49. [CrossRef]

8. Li, X.Y.; Hooft van Huijsduijnen, R.; Mantovani, R.; Benoist, C.; Mathis, D. Intron-exon organization of the NF-Y genes. Tissue-specific splicing modifies an activation domain. J. Biol. Chem. 1992, 267, 8984-8990.

9. Maity, S.N.; de Crombrugghe, B. Biochemical analysis of the B subunit of the heteromeric CCAAT-binding factor. A DNA-binding domain and a subunit interaction domain are specified by two separate segments. J. Biol. Chem. 1992, 267, 8286-8292.

10. Siefers, N.; Dang, K.K.; Kumimoto, R.W.; Bynum, W.E.T.; Tayrose, G.; Holt, B.F. Tissue-specific expression patterns of Arabidopsis NF-Y transcription factors suggest potential for extensive combinatorial complexity. Plant Physiol. 2009, 149, 625-641. [CrossRef]

11. Yang, W.; Lu, Z.; Xiong, Y.; Yao, J. Genome-wide identification and co-expression network analysis of the OsNF-Y gene family in rice. Crop J. 2017, 5, 21-31. [CrossRef]

12. Panahi, B.; Mohammadi, S.A.; Ruzicka, K.; Abbasi Holaso, H.; Zare Mehrjerdi, M. Genome-wide identification and co-expression network analysis of nuclear factor-Y in barley revealed potential functions in salt stress. Physiol. Mol. Biol. Plants 2019, 25, 485-495. [CrossRef] [PubMed]

13. Mai, Y.; Shui, L.; Huo, K.; Niu, J. Genome-wide characterization of the NUCLEAR FACTOR-Y (NF-Y) family in Citrus grandis identified CgNF-YB9 involved in the fructose and glucose accumulation. Genes Genomics 2019, 41, 1341-1355. [CrossRef] [PubMed]

14. Li, M.; Li, G.; Liu, W.; Dong, X.; Zhang, A. Genome-wide analysis of the NF-Y gene family in peach (Prunus persica L.). BMC Genomics 2019, 20, 612. [CrossRef]

15. Wang, Y.; Xu, W.; Chen, Z.; Han, B.; Haque, M.E.; Liu, A. Gene structure, expression pattern and interaction of Nuclear Factor-Y family in castor bean (Ricinus communis). Planta 2018, 247, 559-572. [CrossRef]

16. Wang, P.; Zheng, Y.; Guo, Y.; Chen, X.; Sun, Y.; Yang, J.; Ye, N. Identification, expression, and putative target gene analysis of nuclear factor-Y (NF-Y) transcription factors in tea plant (Camellia sinensis). Planta 2019, 250, 1671-1686. [CrossRef]

17. Maheshwari, P.; Kummari, D.; Palakolanu, S.R.; Nagasai Tejaswi, U.; Nagaraju, M.; Rajasheker, G.; Jawahar, G.; Jalaja, N.; Rathnagiri, P.; Kavi Kishor, P.B. Genome-wide identification and expression profile analysis of nuclear factor $Y$ family genes in Sorghum bicolor L. (Moench). PLoS ONE 2019, 14, e0222203. [CrossRef]

18. Yan, H.; Wu, F.; Jiang, G.; Xiao, L.; Li, Z.; Duan, X.; Jiang, Y. Genome-wide identification, characterization and expression analysis of NF-Y gene family in relation to fruit ripening in banana. Posthar. Biol. Tec. 2019, 151, 98-110. [CrossRef]

19. He, X.; Liu, G.; Li, B.; Xie, Y.; Wei, Y.; Shang, S.; Tian, L.; Shi, H. Functional analysis of the heterotrimeric NF-Y transcription factor complex in cassava disease resistance. Ann. Bot. 2020, 124, 1185-1198. [CrossRef]

20. Siriwardana, C.L.; Gnesutta, N.; Kumimoto, R.W.; Jones, D.S.; Myers, Z.A.; Mantovani, R.; Holt, B.F. NUCLEAR FACTOR Y, subunit A (NF-YA) proteins positively regulate flowering and act through FLOWERING LOCUS T. PLoS Genet. 2016, 12, e1006496. [CrossRef]

21. Hwang, Y.H.; Kim, S.K.; Lee, K.C.; Chung, Y.S.; Lee, J.H.; Kim, J.K. Functional conservation of rice OsNF-YB/YC and Arabidopsis AtNF-YB/YC proteins in the regulation of flowering time. Plant Cell Rep. 2016, 35, 857-865. [CrossRef]

22. Liu, X.; Hu, P.; Huang, M.; Tang, Y.; Li, Y.; Li, L.; Hou, X. The NF-YC-RGL2 module integrates GA and ABA signalling to regulate seed germination in Arabidopsis. Nat. Commun. 2016, 7, 12768. [CrossRef]

23. Tang, Y.; Liu, X.; Liu, X.; Li, Y.; Wu, K.; Hou, X. Arabidopsis NF-YCs mediate the light-controlled hypocotyl elongation via modulating histone acetylation. Mol. Plant 2017, 10, 260-273. [CrossRef] [PubMed]

24. Myers, Z.A.; Kumimoto, R.W.; Siriwardana, C.L.; Gayler, K.K.; Risinger, J.R.; Pezzetta, D.; Holt Iii, B.F. NUCLEAR FACTOR Y, Subunit C (NF-YC) transcription factors are positive regulators of photomorphogenesis in Arabidopsis thaliana. PLoS Genet. 2016, 12, e1006333. [CrossRef] [PubMed]

25. Ballif, J.; Endo, S.; Kotani, M.; MacAdam, J.; Wu, Y. Over-expression of HAP3b enhances primary root elongation in Arabidopsis. Plant Physiol. Biochem. 2011, 49, 579-583. [CrossRef] [PubMed] 
26. Sorin, C.; Declerck, M.; Christ, A.; Blein, T.; Ma, L.; Lelandais-Briere, C.; Njo, M.F.; Beeckman, T.; Crespi, M.; Hartmann, C. A miR169 isoform regulates specific NF-YA targets and root architecture in Arabidopsis. New Phytol. 2014, 202, 1197-1211. [CrossRef]

27. Xu, J.J.; Zhang, X.F.; Xue, H.W. Rice aleurone layer specific OsNF-YB1 regulates grain filling and endosperm development by interacting with an ERF transcription factor. J. Exp. Bot. 2016, 67, 6399-6411. [CrossRef]

28. Bai, A.N.; Lu, X.D.; Li, D.Q.; Liu, J.X.; Liu, C.M. NF-YB1-regulated expression of sucrose transporters in aleurone facilitates sugar loading to rice endosperm. Cell Res. 2016, 26, 384-388. [CrossRef]

29. Xiong, Y.; Ren, Y.; Li, W.; Wu, F.; Yang, W.; Huang, X.; Yao, J. NF-YC12 is a key multi-functional regulator of accumulation of seed storage substances in rice. J. Exp. Bot. 2019, 70, 3765-3780. [CrossRef]

30. Su, H.; Cao, Y.; Ku, L.; Yao, W.; Cao, Y.; Ren, Z.; Dou, D.; Wang, H.; Ren, Z.; Liu, H.; et al. Dual functions of ZmNF-YA3 in photoperiod-dependent flowering and abiotic stress responses in maize. J. Exp. Bot. 2018, 69, 5177-5189. [CrossRef]

31. Yang, M.; Zhao, Y.; Shi, S.; Du, X.; Gu, J.; Xiao, K. Wheat nuclear factor Y (NF-Y) B subfamily gene TaNF-YB3;l confers critical drought tolerance through modulation of the ABA-associated signaling pathway. Plant Cell Tiss. Organ Cult. 2017, 128, 97-111. [CrossRef]

32. Zhou, Y.; Liu, S.; Yang, Z.; Yang, Y.; Jiang, L.; Hu, L. CsCAT3, a catalase gene from Cucumis sativus, confers resistance to a variety of stresses to Escherichia coli. Biotechnol. Biotec. Eq. 2017, 31, 886-896. [CrossRef]

33. Yang, Y.; Ahammed, G.J.; Wan, C.; Liu, H.; Chen, R.; Zhou, Y. Comprehensive analysis of TIFY transcription factors and their expression profiles under jasmonic acid and abiotic stresses in watermelon. Int. J. Genom. 2019, 2019, 6813086. [CrossRef] [PubMed]

34. Wang, Y.; Zhang, Y.; Zhou, R.; Dossa, K.; Yu, J.; Li, D.; Liu, A.; Mmadi, M.A.; Zhang, X.; You, J. Identification and characterization of the bZIP transcription factor family and its expression in response to abiotic stresses in sesame. PLoS ONE 2018, 13, e0200850. [CrossRef] [PubMed]

35. Kumar, S.; Stecher, G.; Tamura, K. MEGA7: Molecular evolutionary genetics analysis version 7.0 for bigger datasets. Mol. Biol. Evol. 2016, 33, 1870-1874. [CrossRef]

36. Li, Z.; Zhang, Z.; Yan, P.; Huang, S.; Fei, Z.; Lin, K. RNA-Seq improves annotation of protein-coding genes in the cucumber genome. BMC Genomics 2011, 12, 540. [CrossRef]

37. Chen, C.; Chen, H.; He, Y.; Xia, R. TBtools, a Toolkit for Biologists integrating various biological data handling tools with a user-friendly interface. bioRxiv 2018, 289660.

38. Zhou, Y.; Li, G.; Zhang, L.; Xu, J.; Hu, L.; Jiang, L.; Liu, S. Comprehensive genomic analysis and expression profiling of the BTB and TAZ (BT) genes in cucumber (Cucumis sativus L.). Czech J. Genet. Plant Breed. 2020, 56, 15-23. [CrossRef]

39. Hackenberg, D.; Wu, Y.; Voigt, A.; Adams, R.; Schramm, P.; Grimm, B. Studies on differential nuclear translocation mechanism and assembly of the three subunits of the Arabidopsis thaliana transcription factor NF-Y. Mol. Plant 2012, 5, 876-888. [CrossRef]

40. Lu, P.; Yu, S.; Zhu, N.; Chen, Y.R.; Zhou, B.; Pan, Y.; Tzeng, D.; Fabi, J.P.; Argyris, J.; Garcia-Mas, J.; et al. Genome encode analyses reveal the basis of convergent evolution of fleshy fruit ripening. Nat. Plants 2018, 4, 784-791. [CrossRef]

41. Feng, Z.J.; He, G.H.; Zheng, W.J.; Lu, P.P.; Chen, M.; Gong, Y.M.; Ma, Y.Z.; Xu, Z.S. Foxtail millet NF-Y families: Genome-wide survey and evolution analyses identified two functional genes important in abiotic stresses. Front. Plant Sci. 2015, 6, 1142. [CrossRef] [PubMed]

42. Quan, S.; Niu, J.; Zhou, L.; Xu, H.; Ma, L.; Qin, Y. Identification and characterization of NF-Y gene family in walnut (Juglans regia L.). BMC Plant Biol. 2018, 18, 255. [CrossRef] [PubMed]

43. Chu, H.D.; Nguyen, K.H.; Watanabe, Y.; Le, D.T.; Pham, T.L.T.; Mochida, K.; Tran, L.P. Identification, structural characterization and gene expression analysis of members of the Nuclear Factor-Y family in chickpea (Cicer arietinum L.) under dehydration and abscisic acid treatments. Int. J. Mol. Sci. 2018, 19, 3290. [CrossRef] [PubMed]

44. Kumimoto, R.W.; Adam, L.; Hymus, G.J.; Repetti, P.P.; Reuber, T.L.; Marion, C.M.; Hempel, F.D.; Ratcliffe, O.J. The Nuclear Factor Y subunits NF-YB2 and NF-YB3 play additive roles in the promotion of flowering by inductive long-day photoperiods in Arabidopsis. Planta 2008, 228, 709-723. [CrossRef]

45. Kumimoto, R.W.; Zhang, Y.; Siefers, N.; Holt, B.F. NF-YC3, NF-YC4 and NF-YC9 are required for CONSTANS-mediated, photoperiod-dependent flowering in Arabidopsis thaliana. Plant J. 2010, 63, 379-391. [CrossRef] [PubMed] 
46. Gnesutta, N.; Chiara, M.; Bernardini, A.; Balestra, M.; Horner, D.; Mantovani, R. The plant NF-Y DNA matrix in vitro and in vivo. Plants 2019, 8, 406. [CrossRef]

47. Zhang, T.; Zhang, D.; Liu, Y.; Luo, C.; Zhou, Y.; Zhang, L. Overexpression of a NF-YB3 transcription factor from Picea wilsonii confers tolerance to salinity and drought stress in transformed Arabidopsis thaliana. Plant Physiol. Biochem. 2015, 94, 153-164. [CrossRef]

48. Chen, M.; Zhao, Y.; Zhuo, C.; Lu, S.; Guo, Z. Overexpression of a NF-YC transcription factor from bermudagrass confers tolerance to drought and salinity in transgenic rice. Plant Biotechnol. J. 2015, 13, 482-491. [CrossRef]

49. Wu, X.; Shi, H.; Guo, Z. Overexpression of a NF-YC gene results in enhanced drought and salt tolerance in transgenic seashore paspalum. Front. Plant Sci. 2018, 9, 1355. [CrossRef]

50. Sato, H.; Suzuki, T.; Takahashi, F.; Shinozaki, K.; Yamaguchi-Shinozaki, K. NF-YB2 and NF-YB3 have functionally diverged and differentially induce drought and heat stress-specific genes. Plant Physiol. 2019, 180, 1677-1690. [CrossRef]

51. Ma, X.; Li, C.; Wang, M. Wheat NF-YA10 functions independently in salinity and drought stress. Bioengineered 2015, 6, 245-247. [CrossRef] [PubMed]

(C) 2020 by the authors. Licensee MDPI, Basel, Switzerland. This article is an open access article distributed under the terms and conditions of the Creative Commons Attribution (CC BY) license (http://creativecommons.org/licenses/by/4.0/). 